\title{
Confinement effects in a guided-wave interferometer with millimeter-scale arm separation
}

\author{
J. H. T. Burke, B. Deissler, K. J. Hughes and C. A. Sackett \\ University of Virginia, Dept. of Physics, Charlottesville, VA 22904
}

\begin{abstract}
Guided-wave atom interferometers measure interference effects using atoms held in a confining potential. In one common implementation, the confinement is primarily two-dimensional, and the atoms move along the nearly free dimension under the influence of an off-resonant standing wave laser beam. In this configuration, residual confinement along the nominally free axis can introduce a phase gradient to the atoms that limits the arm separation of the interferometer. We experimentally investigate this effect in detail, and show that it can be alleviated by having the atoms undergo a more symmetric motion in the guide. This can be achieved by either using additional laser pulses or by allowing the atoms to freely oscillate in the potential. Using these techniques, we demonstrate interferometer measurement times up to $72 \mathrm{~ms}$ and arm separations up to $0.42 \mathrm{~mm}$ with a well controlled phase, or times of $0.91 \mathrm{~s}$ and separations of $1.7 \mathrm{~mm}$ with an uncontrolled phase.
\end{abstract}




\section{Introduction}

Atom interferometry is a striking example of the particle-wave duality inherent in quantum mechanics. Beyond this, however, it has proven to be a practical and useful technique for several types of precision measurements [1]. Traditional atom interferometers use atoms moving freely through space, with only occasional manipulation by external fields to control their trajectory. With the development of laser cooling techniques and Bose-Einstein condensation, however, it has become possible to implement "guided-wave" atom interferometers, in which an applied potential directs the atomic motion at all times [2-7]. A useful analogy is the comparison between an optical interferometer constructed using free beams manipulated by mirrors, versus one using light confined in optical fibres. The advantages of guided-wave atom interferometry include the ability to incorporate more complex trajectories, and also to support the atoms against gravity over long measurement periods. It is hoped that these advantages will eventually allow guided-wave interferometers to exceed the capabilities of free-space devices. Guided-wave atom interferometry presents several challenges, however, since it is critical that the guiding fields impart no uncontrolled quantum phase to the wavepackets.

One limitation that is particularly important for interferometers using larger arm separation is the effect of longitudinal variations in the guiding potential. In several experiments to date $[4,6,8]$, the "guide" is really an elongated harmonic trap, with atomic wave packets moving along the weak axis of the trap. The nonuniform potential imparts a spatially varying phase shift to the packets, so that different parts of the packet interfere with different phases and the overall visibility of the interference is degraded. In this paper, we explore this phenomenon in detail by experimentally measuring the phase gradients imposed and confirming their relation to the visibility. We also demonstrate that the effect can be much reduced by using more symmetric trajectories for the atomic packets. In this way, packet separations of up to $0.42 \mathrm{~mm}$ have been achieved with controllable overall phase, and interference is still observed for separations of $1.7 \mathrm{~mm}$. In the latter case, however, environmental noise effects cause the phase to fluctuate randomly from one measurement to the next.

One figure of merit for an interferometer is the time integral of the wave packet separation, which we denote $\chi$. This gives directly the accumulated phase for measurement of a linear gradient field such as gravity. To put the results in context, free space interferometers reach $\chi \approx 500 \mu \mathrm{m} \cdot \mathrm{s}[9,10]$. Previous results for our experiment were limited to $\chi=6 \mu \mathrm{m} \cdot \mathrm{s}$ [6], and other approaches have yielded $\chi \approx 2 \mu \mathrm{m} \cdot \mathrm{s}$ $[7,11]$. The improvements reported here yield $\chi=15 \mu \mathrm{m} \cdot \mathrm{s}$ with controllable phase, and $850 \mu \mathrm{m} \cdot \mathrm{s}$ with uncontrolled phase.

Our interferometer has been described in detail in Ref. [6,12], and the basic

operation is illustrated in Fig. 1(a). A Bose-Einstein condensate of $3 \times 10^{4}{ }^{87} \mathrm{Rb}$ atoms is prepared in the $F=2, m_{F}=2$ hyperfine state and held in a time-orbiting potential waveguide with harmonic confinement at frequencies $\left(\omega_{x}, \omega_{y}, \omega_{z}\right) \approx 2 \pi \times(6.0,1.1,3.3) \mathrm{Hz}$. 

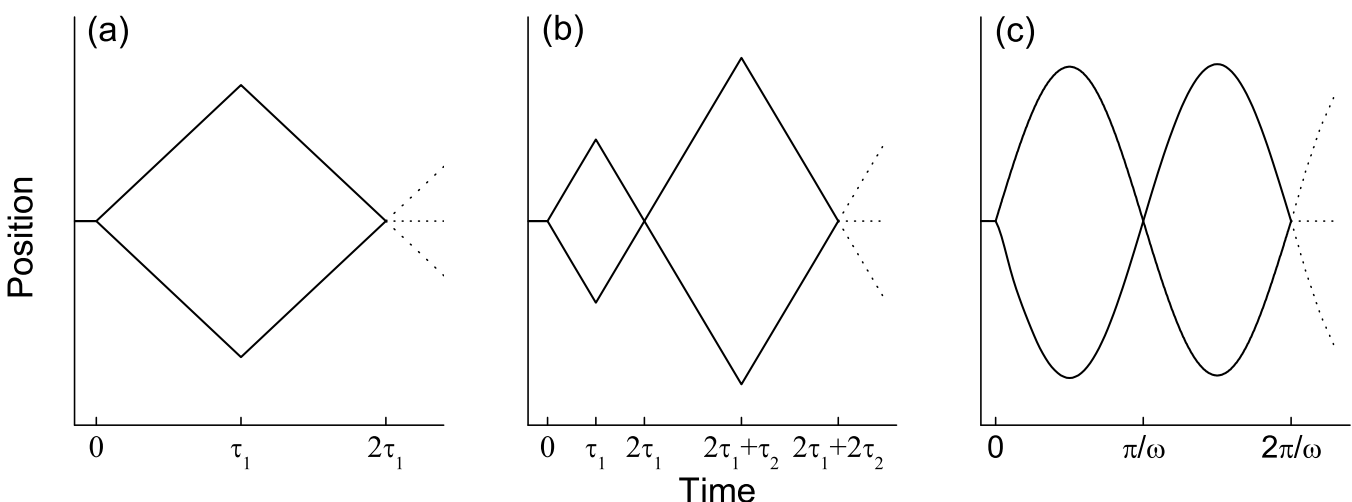

Figure 1. Wave packet trajectories for various interferometer configurations. (a) Single-sided, with leg duration $\tau_{1}$. (b) Double-sided, with leg duration $\tau_{1}$ for the first half and $\tau_{2}$ for the second half. (c) Free oscillation in a waveguide with harmonic confinement at frequency $\omega$. Note that the time and position scales vary considerably for the different sub-figures.

We note that this confinement is weaker than that used in other comparable experiments. The atoms are manipulated using an off-resonant laser tuned $12 \mathrm{GHz}$ blue of the $5 S_{1 / 2} \leftrightarrow 5 P_{3 / 2}$ principle transition at wavelength $\lambda=780 \mathrm{~nm}$. The beam is reflected to form a standing wave aligned to the $y$-axis of the waveguide. By applying a double pulse of this standing wave [13], atoms at rest in the $|0\rangle$ momentum state can be driven to a superposition of states moving with momentum $p= \pm 2 \hbar k:|0\rangle \rightarrow(|2 \hbar k\rangle+|-2 \hbar k\rangle) / \sqrt{2}$, where $k=2 \pi / \lambda$ is the wavenumber of the standing wave laser. In our case, the moving atoms acquire a speed of $v_{0}=11.7 \mathrm{~mm} / \mathrm{s}$. As described in Ref. [14], this operation can be made very precise. After this 'splitting' is complete, the packets propagate away from each other in the $y$ direction for a time $\tau_{1}$. The standing wave is then applied a second time, with intensity and duration such that the 'reflection' transition $|2 \hbar k\rangle \leftrightarrow|-2 \hbar k\rangle$ is driven. After a total time $2 \tau_{1}$, the packets return to their initial position. A third laser pulse, identical to the first splitting pulse, can then be applied. If there is no phase difference between the two packets of atoms, this final pulse reverses the action of the first, and all the atoms come back to rest. However, if we apply a phase $\theta$ between the packets, some portion of the atoms will continue in the moving states. The fraction of atoms in the rest state, $N_{0} / N$, is given by $\cos ^{2}(\theta / 2)$. In practice, the phase $\theta$ is controlled by adjusting the frequency of the standing wave laser before the final pulse $[6,15]$. We refer to the simple trajectory of Fig. 1(a) as a single-sided interferometer.

In this geometry, it is not difficult to see how the axial confinement can produce a phase gradient across the packets. The packets have a length along the guide of $L$, set by the size of the initial condensate. As the packets move apart, the leading edge of the packet experiences a larger potential energy than the trailing edge, since it is further 
away from the bottom of the potential. This edge therefore develops a larger phase, which continues to accumulate through the entire trajectory. Since the leading edge of one packet interferes with the trailing edge of the other, the phase difference between the packets has a spatial gradient.

This problem was first recognized by Olshanii and Dunjko [16], who used it to explain the limited interferometer performance observed by Wang et al. [4]. Later, Horikoshi and Nakagawa [8] demonstrated an interferometer using a similar geometry and showed that it too was limited to an arm separation consistent with that imposed by the phase gradient effect. In these experiments, interactions between the atomic packets also played a significant role. As the packets separate, different parts of the packets interact for different times. This also induces a phase gradient, which can be comparable to the confinement effect described above. Stickney et al. [17] have suggested that the confinement and interaction gradients can be made to cancel in some cases, allowing the arm separation to be extended.

Due to the weak confinement of our waveguide, our experiment operates in a somewhat different regime, where the effects of interactions are small and the confinement effects dominate. We assert that any interferometer with well-separated arms will necessarily be in this regime, since the interaction phase stops growing once the packets are separated while the confinement effect continues to accelerate. The solution we examine here is to use a more symmetric interferometer geometry, as shown in Fig. 1(b). Here the packets are not immediately recombined when they return to the centre. Instead, they are allowed to continue propagating for another interval $\tau_{2}$, whereupon they are reflected a second time. They finally overlap again and are recombined at total time $2\left(\tau_{1}+\tau_{2}\right)$, as illustrated in Fig 1(b). In the second half of this 'double-sided' interferometer, the leading and trailing edges of the packets exchange roles, so that the confinement phase accumulates with opposite sign. When $\tau_{1}=\tau_{2}$, the phases from the two halves nearly cancel and the arm separation can be significantly extended. We made use of this type of trajectory previously [6], but here we investigate the phase cancellation effect in detail. We also demonstrate an arm separation about twice that previously achieved, due to various improvements in the apparatus [14]. Even larger separations can be obtained using no reflection pulses at all. If the packets are simply allowed to oscillate freely for one period of the harmonic guide potential as in Fig. 1(c), then the two halves of the cycle are entirely symmetric and the phase gradient cancels more precisely. The phase output of this interferometer is currently unpredictable, however, which renders its use impractical.

The remainder of the paper is organized as follows: Section 2 describes the theoretical analysis and experimental investigation of the one-sided 'single-reflect' interferometer. Section 3 discusses the two-sided interferometer. Section 4 presents the results of the free oscillation interferometer, and Section 5 offers a summary and conclusions. 


\section{Single-sided interferometer}

We estimate the phase gradient for our experimental conditions using a simple semiclassical theory. We neglect any internal dynamics of the packets themselves, treating them as rigid bodies. This is justified since the internal motion occurs on the time scale of the confinement frequency $\omega$, and the confinement effects constrain the interferometer time $\tau_{1}$ to $\omega \tau_{1} \ll 1$, as will be seen. Additionally, we assume that the atoms start at rest at the minimum of the trap. Non-zero initial motion of the condensate is not difficult to include, and can change the phase development. However, in our experiment, this effect is small enough to neglect. The potential along the $y$-axis of the guide taken to be

$$
V_{\text {ext }}(y)=\frac{1}{2} m \omega^{2} y^{2}
$$

We define $y_{1}(t)$ and $y_{2}(t)$ to be the positions of the centres of the two packets produced by the splitting operation. Within a packet, the position relative to the centre is labelled $\xi_{1}$ or $\xi_{2}$.

The phase acquired by packet 1 over the total time of the interferometer is

$$
\phi_{1}\left(\xi_{1}\right)=\frac{1}{\hbar} \int_{0}^{2 \tau_{1}} V_{\text {ext }}\left(y_{1}+\xi_{1}\right) d t=\frac{m \omega^{2}}{2 \hbar} \int_{0}^{2 \tau_{1}}\left[y_{1}(t)+\xi_{1}\right]^{2} d t
$$

Before the reflection pulse, the packet trajectory is given by

$$
y_{1}(t)=\frac{v_{0}}{\omega} \sin (\omega t) \quad\left(0<t<\tau_{1}\right)
$$

At time $\tau_{1}$, the reflection pulse provides a velocity kick of $-2 v_{0}$, so the subsequent trajectory is

$$
\begin{aligned}
y_{1}(t)=\frac{v_{0}}{\omega} & \sin \left(\omega \tau_{1}\right) \cos \left[\omega\left(t-\tau_{1}\right)\right] \\
& +\frac{v_{0}}{\omega}\left[\cos \left(\omega \tau_{1}\right)-2\right] \sin \left[\omega\left(t-\tau_{1}\right)\right] \quad\left(\tau_{1}<t<2 \tau_{1}\right) .
\end{aligned}
$$

The second packet follows a similar trajectory with $v_{0}$ replaced by $-v_{0}$ everywhere.

Notice that the 'reflect' pulse really is a momentum kick and not a true reflection. This has an effect that will be important in Section 3. As the packets move to higher potential they slow down, with packet 1 reaching a velocity $v=v_{0}-\delta v$. The reflection pulse alters this to $-v_{0}-\delta$, so the packet is then moving faster than $v_{0}$. As the packet returns to the origin, it accelerates further, to approximately $v=-v_{0}-2 \delta v$. Because of this, at time $2 \tau_{1}$ the two packets are not precisely overlapped, but have moved past one another [17]. This can be corrected by modifying the length of time between reflect and recombination, but in our experiment the effect is negligible for the single-sided interferometer and we have $y_{1}\left(2 \tau_{1}\right) \approx y_{2}\left(2 \tau_{1}\right)$, so that $\xi_{1} \approx \xi_{2} \equiv \xi$.

Evaluation of the integral in (2) gives

$$
\phi=\phi_{1}(\xi)-\phi_{2}(\xi)=-4 k \xi\left[\cos \left(2 \omega \tau_{1}\right)-2 \cos \left(\omega \tau_{1}\right)+1\right]
$$

using $v_{0}=2 \hbar k / m$. For $\omega \tau_{1} \ll 1$, the phase is approximately $4 k \xi\left(\omega \tau_{1}\right)^{2}$. A smaller effect comes from the interaction between the packets while they are still overlapped. 
To include this, we treat the system as one-dimensional, so that the initial condensate satisfies the Gross-Pitaevskii equation

$$
i \hbar \frac{\partial}{\partial t} \Psi(y, t)=\left[-\frac{\hbar^{2}}{2 m} \frac{\partial^{2}}{\partial^{2} y}+V_{\text {ext }}(y)+g_{1 D}|\psi(y, t)|^{2}\right] \Psi(y, t),
$$

where $g_{1 D}$ is the one dimensional coupling constant [18]. The actual experiment is not accurately in the one-dimensional limit, but it provides a convenient approximation for what is anyway a modest correction. In the Thomas-Fermi limit the number density is

$$
n_{1 D}(y)= \begin{cases}\frac{\mu}{g_{1 D}}\left(1-\frac{y^{2}}{L^{2}}\right) & -L<y<L \\ 0 & \text { otherwise }\end{cases}
$$

for chemical potential $\mu$ and condensate half-length $L=\sqrt{2 \mu / m \omega^{2}}$. In the experiment, $L=55 \mu \mathrm{m}$, implying $\mu=2 \pi \hbar \times 15 \mathrm{~Hz}$. Equation (7) can be normalized to the total number of atoms $N$ using $\int n_{1 D} d y=N$, which yields $\mu / g_{1 D}=3 N / 4 L$.

The interaction potential between the packets is $U_{\text {int }}=2 g_{1 D} n_{1 D}$, where the factor of two comes from the exchange effect; however once split the number density is reduced by a factor of two, leaving

$$
U_{\text {int }}\left(\xi_{1}\right)= \begin{cases}\mu\left(1-\frac{\xi_{2}^{2}}{L^{2}}\right) & -L<\xi_{2}<L \\ 0 & \text { otherwise }\end{cases}
$$

An atom at position $\xi_{1}$ in the first packet will experience the potential due to atoms at $\xi_{2}$ in the second packet. For times small compared to $\omega^{-1}$ we relate two positions by approximating the packet centres as $y_{1,2}= \pm v_{0} t$, so that position $\xi_{1}$ in the first packet coincides with $\xi_{2}-2 v_{0} t$ in the other packet. The phase difference due to interactions is then

$$
\phi_{\text {int }}(\xi)=\frac{1}{\hbar} \int_{0}^{2 \tau_{1}}\left[U_{\text {int }}\left(\xi-2 v_{0} t\right)-U_{\text {int }}\left(\xi+2 v_{0} t\right)\right] d t
$$

This can be evaluated as

$$
\phi_{\text {int }}= \begin{cases}8 k \xi\left(\omega \tau_{1}\right)^{2} & \text { if } \xi<L-2 v \tau_{1} \\ \frac{m \omega^{2}}{\hbar v_{0}} \xi(L-\xi)^{2} & \text { if } \xi>L-2 v \tau_{1}\end{cases}
$$

In our experiment, the packets separate at $\tau_{1}>L / v_{0}=4.7 \mathrm{~ms}$. For times larger than this, the second expression applies at all positions in the packet.

When the packets are finally recombined, the fraction of atoms brought to rest is $[1+\cos (\theta+\phi)] / 2$, where $\theta$ is an applied phase and $\phi$ is the confinement phase calculated above. Since $\phi$ depends on position, the final packet density will exhibit a sinusoidal modulation. This can be experimentally observed as follows: The recombination pulse generally produces three packets, at velocities of $0, v_{0}$ and $-v_{0}$. After the pulse, we wait some time for these packets to separate and then use a resonant probe beam to take an absorption image. Figure 2 shows examples of the images obtained. Image (a) shows the initial condensate, with no gradient. Images (b) and (c) show packets obtained 


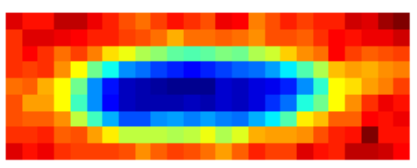

(a)

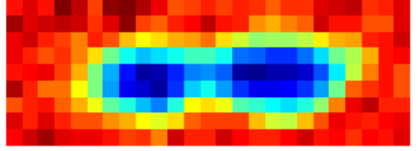

(b)

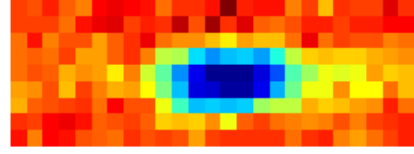

(c)

Figure 2. (colour) Absorption images of wave packets used in the condensate interferometer. (a) The condensate distribution, before the interferometer operation. (b) A packet obtained at the output of the interferometer, showing the effect of a phase gradient. (c) Another output packet, showing the effect of shifting the overall phase of the interferometer by $\pi$.

after the interferometer sequence, with overall phase differing by $\pi$. Both images show a modulation of the atomic density, with suppression at either the centre (a) or edges (b) of the packet.

To analyze such images, we integrated the absorption signal in the direction transverse to the interferometer axis, thereby obtaining a one-dimensional profile. We then simultaneously fit up to 12 packets (four images of three packets each) to a modulated Thomas-Fermi function with a common gradient $G$ and fixed width $L$ :

$$
A(y)=A_{0}+B \sin ^{2}\left[G\left(y-y_{0}\right)+\theta\right] \times \min \left[0,1-\left(\frac{y-y_{0}}{L}\right)^{2}\right] .
$$

Each of the images in a set is taken with the same experimental conditions except for the applied phase $\theta$, which is varied. From the fit, we acquire $G=\frac{1}{2}|d \phi / d \xi|$. For gradients comparable to $1 / L$ or smaller, the fits become less reliable as there is little modulation to observe.

Figure 3(a) shows the measured and calculated gradients as functions of the total measurement time $2 \tau_{1}$. The piecewise behaviour for small $\tau_{1}$ is caused by the interaction effect. The gradient from interactions depends on position in the packet; here we plot the gradient at the centre $\xi=0$. The measured gradients agree well with the theoretical expectation.

Normally, we operate the interferometer by measuring the total number of atoms in each of the three packets to determine $N_{0} / N$. This signal can be evaluated as

$$
\frac{N_{0}}{N}=\frac{1}{2}\left[1+\int_{-L}^{L} n_{1 D}(\xi) \cos [\theta+\phi(\xi)] d \xi\right] .
$$

Using the symmetry of $\phi$ this reduces to

$$
\frac{N_{0}}{N}=\frac{1}{2}\left[1+\cos (\theta) \frac{3}{4 L} \int_{-L}^{L}\left(1-\frac{\xi^{2}}{L^{2}}\right) \cos \phi d \xi\right],
$$

from which the visibility of the interference is seen to be

$$
V=\frac{3}{4 L} \int_{-L}^{L}\left(1-\frac{\xi^{2}}{L^{2}}\right) \cos \phi d \xi
$$

Experimentally, the visibility and its uncertainty are measured by scanning $\theta$ and fitting the resulting signal to the form $(1+V \cos \theta) / 2$. Figure $3(\mathrm{~b})$ shows a comparison of 

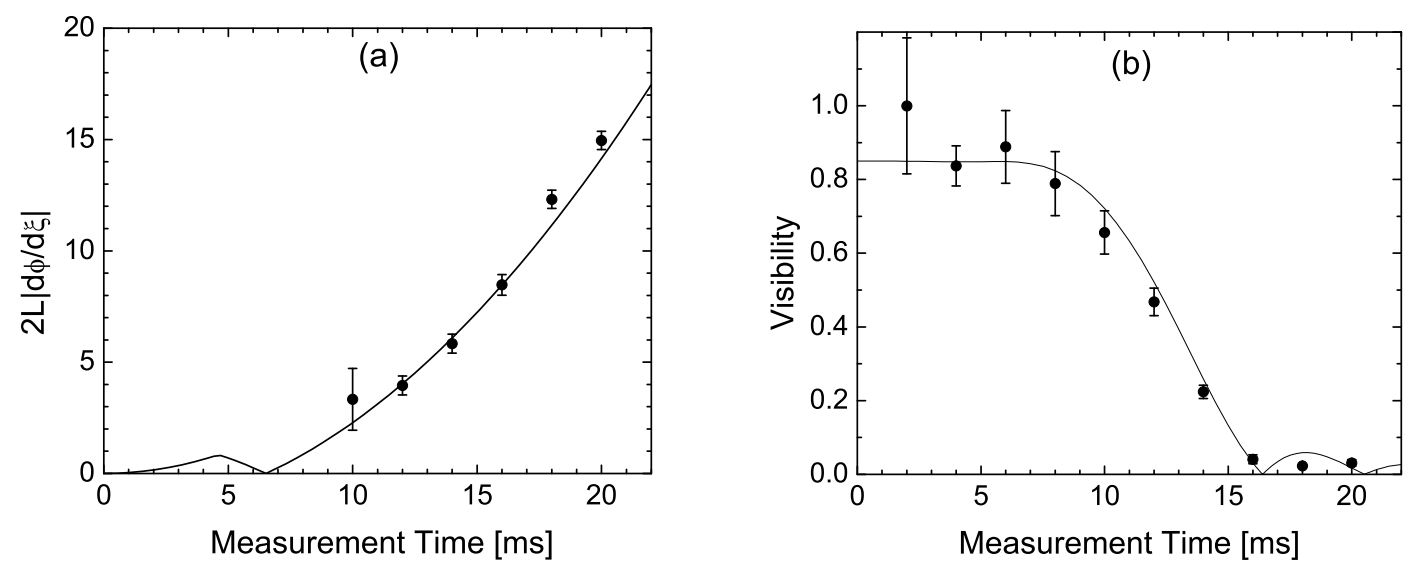

Figure 3. (a) Magnitude of the phase gradient for a single-sided interferometer of total duration $2 \tau_{1}$. Points show the experimental measurements, with error bars determined by the fitting procedure. The curve shows the results of the calculation described in the text, with the gradient scaled to the length of the packet, $2 L$. (b) Visibility of the single-sided interferometer, as a function of total duration. Points show the measured values, while the curve shows the theoretical prediction. The prediction has been scaled by 0.85 to account for experimental imperfections that are expected to be independent of duration.

the measured and predicted visibility curves. Even at short times, we obtain typical visibilities of about 0.85 , due to imperfections in the standing wave operations and other experimental effects. We therefore scale the theoretical curve by this factor. Again, good agreement is obtained.

The figure shows that the visibility is reduced by one half at $2 \tau_{1} \approx 12 \mathrm{~ms}$, corresponding to a gradient of 4 radians across the full packet. At this time, the centres of the clouds are $120 \mu \mathrm{m}$ apart, meaning the packets are just separated. This makes it difficult, for instance, to apply a field to one packet without influencing the other, and yields a figure of merit $\chi$ of only $0.07 \mu \mathrm{m} \cdot \mathrm{s}$. It is possible to operate the interferometer with larger phase gradients, by considering the density profile as above and taking the fit phase $\theta$ in Eq. 11 as the interferometer output. However, this introduces considerable noise in practice because of imaging imperfections such as the limited spatial resolution.

The results of this section illustrate clearly that the operation of the single-sided interferometer is limited by phase gradient effects.

\section{Double-sided interferometer}

The confinement phase gradient is alleviated in a double-sided interferometer, where the gradients from the two halves tend to cancel out. To analyze this situation, consider an asymmetric interferometer with leg duration $\tau_{1}$ in the first half and $\tau_{2}$ in the second 

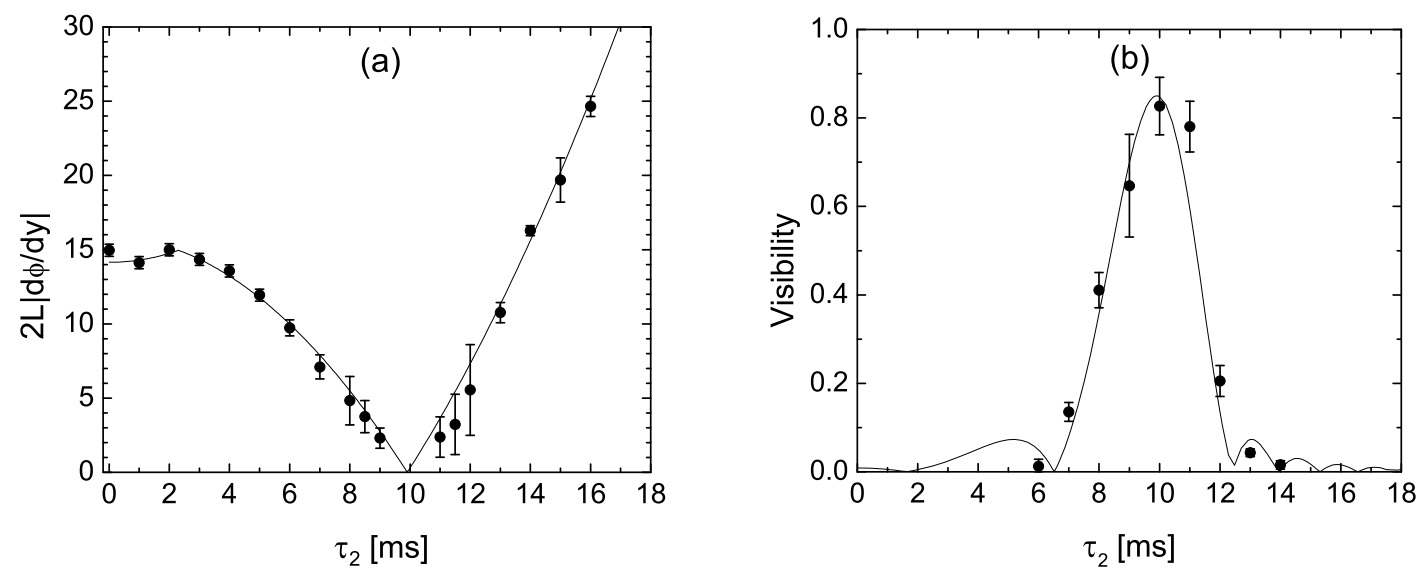

Figure 4. (a) Phase gradient observed in an asymmetric double-sided interferometer. The first half of the trajectory used leg time $\tau_{1}$ and the second half $\tau_{2}$. Here $\tau_{1}=10 \mathrm{~ms}$ and $\tau_{2}$ is varied. When $\tau_{2}=\tau_{1}$, the phase gradient is nearly eliminated. (b) Interferometer visibility as $\tau_{2}$ is varied. For both plots, data points show the experimental results and the curves show the model calculation. In (b), the calculation is scaled by 0.85 as in Fig. 3 .

half, as in Fig. 1(b). The calculation of (2) is readily extended to this case, yielding

$$
\phi=4 k \xi\left\{\cos \left[2 \omega\left(\tau_{1}+\tau_{2}\right)\right]-2 \cos \left[\omega\left(\tau_{1}+2 \tau_{2}\right)\right]+2 \cos \left(\omega \tau_{2}\right)-1\right\} .
$$

In the case $\tau_{1}=\tau_{2}=\tau$, this gives to leading order a gradient $d \phi / d \xi=16 k(\omega \tau)^{4}$, suppressed by a factor of $(2 \omega \tau)^{2}$ compared to the single-sided interferometer. The gradient due to interactions can be calculated as well, but here the maximum gradient obtained for separated arms becomes so small to be nearly negligible. For both sources of gradient, the cancellation is not perfect because the packet velocities during the two halves are not exactly equal, as discussed above. Since the velocity correction $\delta$ is on the order of $v_{0}(\omega \tau)^{2}$, the scaling of the residual gradient is sensible.

We observe the cancellation effect by fixing $\tau_{1}=10 \mathrm{~ms}$ and varying $\tau_{2}$. The results are seen in Fig. 4(a), where the gradient becomes unobservably small when $\tau_{2}=\tau_{1}$. The effect of interactions is seen at $\tau_{2}<2 \mathrm{~ms}$. Figure 4(b) shows how the resulting visibility is recovered. We note that the cancellation effect is very much analogous to the spin echo phenomenon known in magnetic resonance studies.

Using this approach, the measurement time of the interferometer can be significantly extended, as seen in Fig. 5. The data agree reasonably well with the predicted values, but in this case some caution in the interpretation is warranted. At such long times, the approximation that the internal dynamics of the packet is negligible is no longer well justified. The period of oscillation in the tightest direction of the guide is only $166 \mathrm{~ms}$, and interactions will couple motions in the transverse and longitudinal directions. Because of this, the length of the condensate can be expected to vary on these time scales, which will alter the phase gradient. Given that the predicted gradient is 


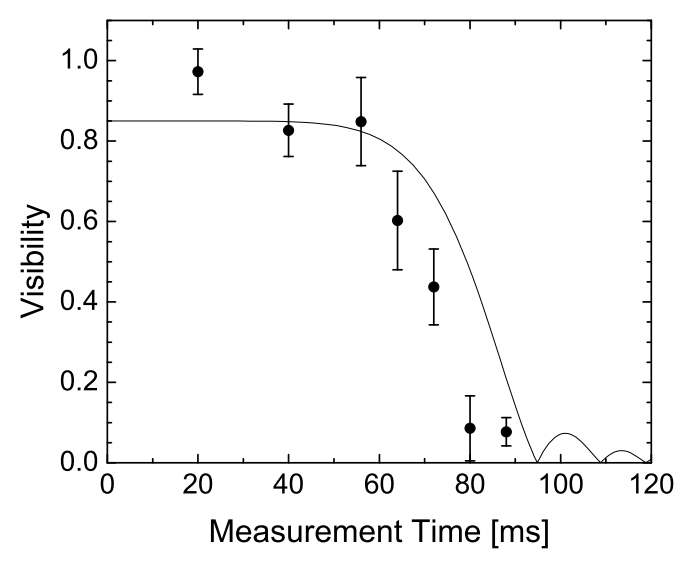

Figure 5. Visibility of symmetric double-sided interferometer, as a function of the total measurement time $T=4 \tau$. Data points show the experimental results and the curve shows the model prediction, scaled by 0.85 as in Fig. 3.

already the difference of two relatively large values, even a small effect from the internal dynamics could be significant. Accurate calculation of the packet dynamics would require the numerical solution of the full three-dimensional Gross-Pitaevskii equation.

Experimental evidence of the inadequacy of the simple theory presented in Section 2 is that as the visibility of the double-sided interferometer falls to zero, images of the atoms do not exhibit clear gradients like those seen in Fig. 2. Instead, the recombination appears to be uniform. A possible explanation is that transverse gradients develop along with the longitudinal ones, since the absorption imaging technique averages over phase variations that are parallel to the probe beam. Visibility will also be lost if the standing-wave laser is misaligned, so that the packets are not spatially overlapped when the recombination pulse is applied. We ruled this out by observing the packets along two axes to verify their alignment in all three dimensions. A third possibility is overall phase noise such as that resulting from vibrations of the mirror producing the standing wave. This would cause the interferometer signal to differ from shot to shot and reduce the visibility upon averaging. However, we do not observe the shot-to-shot variations that would characterize such noise. For instance, at at total measurement time $T=100 \mathrm{~ms}$, the deviation in $N_{0} / N$ from one experiment to the next is only $1 \%$.

These experiments demonstrate that the double-sided interferometer extends the useful measurement time of the interferometer by a factor of six compared to the singlesided case. This improves the arm separation by a factor of three, allowing the packets to be well separated and individually accessible. We note that a double-sided interferometer is not suited for some types of measurements. In a measurement of gravity, for instance, the gravitational phase of the two arms would cancel just as the confinement phase does. However, many other measurements remain possible, including the Sagnac phase in a loop geometry and any case where the effect to be measured can be applied at a definite 
time. We have recently used this technique to interferometrically measure the dynamic polarizability of ${ }^{87} \mathrm{Rb}[19]$.

\section{Free-oscillation interferometer}

Unfortunately, the same trick cannot be repeated to extend the arm separation even further. We theoretically considered a 'four-sided' interferometer, where the packets pass through each other three times before being recombined. This did reduce the phase gradient, but only by a factor of four, rather than by a power of $\omega \tau$. Given the $\tau^{4}$ scaling of the gradient, this would provide only a modest increase in arm separation. At the same time, imperfections in the reflection operations begin to have a significant effect, so we did not pursue this in the experiment.

Instead, we implemented an interferometer in which no reflection pulses are used. The packets are split, allowed to complete a full natural oscillation in the guide, and then recombined as shown in Fig. 1(c). Insofar as the guide potential is harmonic, the trajectories for the two half-cycles will be symmetric and any phase gradients should cancel precisely. However, the oscillation period in our guide is $0.9 \mathrm{~s}$. Over such a long time scale, many external noise sources can impart an uncontrolled phase difference to the packets, making the interferometer output effectively random. We were therefore unable to observe a controlled interference signal as in the previous experiments, but rather used the run-to-run variations in the output as a measure of the interference visibility. This technique, including the use of a freely oscillating trajectory, was also demonstrated by Segal et al. [20]. Their results corresponded to a figure of merit $\chi=40 \mu \mathrm{m} \cdot \mathrm{s}$.

For such long measurement times, the alignment of the standing wave beam to the guide axis is critical, to avoid having the packets miss each other in the transverse directions. In Fig. 6, we show the variance of $N_{0} / N$ observed as the beam alignment angle is varied, plotted against the measured packet displacement. The clear peak indicates that interference is present. Assuming the fluctuations represent an underlying distribution $(1+V \cos \theta) / 2$ with random $\theta$, the variance $\Delta^{2}$ is related to the visibility $V$ by $\Delta^{2}=V^{2} / 8$, thus suggesting $V=0.3$. A possible reason for the imperfect visibility is the effect of internal dynamics introducing asymmetry to the two halves of the trajectory. We note that no interference was observed when the atoms were allowed to oscillate for only one half of the axial period.

In this experiment, the maximum arm separation is $1.7 \mathrm{~mm}$. Without control of the overall phase, it is not possible to make use of this arm separation in a practical measurement, but it can be hoped that this is a technical problem that is solvable through various stabilization methods. If so, this type of interferometer would offer an unprecedented arm separation and measurement time. 


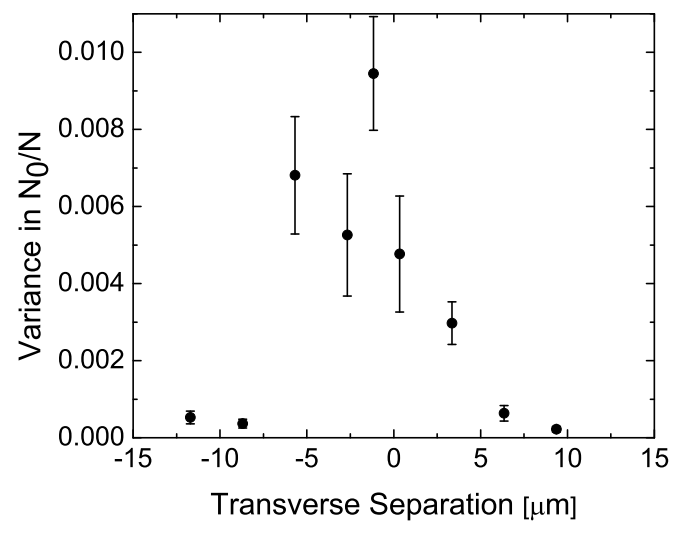

Figure 6. Interference for atoms freely oscillating in the waveguide. The measurement time is $0.9 \mathrm{~s}$, corresponding to a separation of $1.7 \mathrm{~mm}$. The phase of the interference is random, so the variance of the signal measured over several experiments is plotted against the transverse separation of the wave packets at the time of the recombination pulse. The large increase in variance when the packets are overlapped is taken as a signal that interference is present. The error bars represent the error in the variance, estimated as $\Delta^{2} / N$ for variance $\Delta^{2}$ and number of measurements $N$.

\section{Conclusions/Acknowledgments}

We have studied the effect of longitudinal confinement on a guided wave interferometer, under various conditions. It is seen conclusively that for a single-sided interferometer, large phase gradients induced by the confinement potential place a severe limit on the usable arm separation of the the interferometer. The phase gradients were measured and found to agree well with a simple theory in which the internal dynamics of the atomic wave packets are neglected.

The interferometer performance can be significantly improved using a double-sided trajectory, in which each packet experiences each side of the potential. In this case, measurement times of up to $72 \mathrm{~ms}$ and arm separations of $420 \mu \mathrm{m}$ are achieved. An even larger arm separation of $1.7 \mathrm{~mm}$ can be obtained by allowing the packets to complete a full oscillation in the guide, with no imposed reflection pulses. In this case, however, noise effects introduce a random phase shift that prevents the device from being practically useful. When the measurement time of the interferometer becomes comparable to the motional period in the transverse directions of the guide, accurate predictions of performance will likely require a more sophisticated model in which the packet dynamics are included.

One conclusion to be drawn is that axial confinement in a guided wave interferometer is generally a detriment and should be avoided. In a linear interferometer, the axial potential typically comes from the electrical connections at the end of the guide, so simply making the guide longer would improve the flatness [5]. Alternatively, additional current elements could be added to raise the potential in the centre of the 
guide. It is also worth noting that these effects should generally be suppressed in a ring-shaped guide. Even if the potential around the ring is not perfectly uniform, the net phase gradient will be close to zero if the packets are allowed to propagate around the full circumference. In this case no reflection pulses are required, making it similar to the free-oscillation interferometer demonstrated here. Through considerations such as these, we expect that the findings here will be useful for guiding future designs.

We are grateful to J. Stickney and A. Zozulya for useful conversations on these subjects. This work was funded by the U.S. Defense Advanced Research Projects Agency, under grant number W911NF-06-1-0474.

\section{References}

[1] P R Berman, editor. Atom Interferometry. Academic Press, San Diego, 1997.

[2] Y Shin, M Saba, T A Pasquini, W Ketterle, D E Pritchard, and A E Leanhardt. Atom interferometry with Bose-Einstein condensates in a double-well potential. Phys. Rev. Lett., 92:050405, 2004.

[3] T Schumm, S Hofferberth, L M Anderssona, S Wildermuth, S Groth, I Bar-Joseph, J Schmiedmayer, and P Krüger. Matter-wave interferometry in a double well on an atom chip. Nat. Phys., 1:57, 2005.

[4] Y J Wang, D Z Anderson, V M Bright, E A Cornell, Q Diot, T Kishimoto, M Prentiss, R A Saravanan, S R Segal, and S Wu. Atom Michelson interferometer on a chip using a BoseEinstein condensate. Phys. Rev. Lett., 94:090405, 2005.

[5] S Wu, E J Su, and M Prentiss. Time domain deBroglie wave interferometry along a magnetic guide. Euro. Phys. J. D, 35:111, 2005.

[6] O Garcia, B Deissler, K J Hughes, J M Reeves, and C A Sackett. Bose-Einstein-condensate interferometer with macroscopic arm separation. Phys. Rev. A, 74:031601(R), 2006.

[7] G-B Jo, Y Shin, S Will, T A Pasquini, M Saba, W Ketterle, D E Pritchard, M Vengalattore, and M Prentiss. Long phase coherence time and number squeezing of two Bose-Einstein condensates on an atom chip. Phys. Rev. Lett., 98:030407, 2007.

[8] M Horikoshi and K Nakagawa. Dephasing due to atom-atom interaction in a waveguide interferometer using a Bose-Einstein condensate. Phys. Rev. A, 74:031602(R), 2006.

[9] A Peters, K Y Chung, and S Chu. High-precision gravity measurements using atom interferometry. Metrologia, 38:25, 2001.

[10] J M McGuirk, G T Foster, J B Fixler, M J Snadden, and M A Kasevich. Sensitive absolute-gravity gradiometry using atom interferometry. Phys. Rev. A, 65:033608, 2002.

[11] E Su, S Wu, and M Prentiss. Atom interferometry using wavepackets with constant spatial separations. arXiv: physics/0701018, 2007.

[12] J M Reeves, O Garcia, B Deissler, K L Baranowski, K J Hughes, and C A Sackett. A time-orbiting potential trap for Bose-Einstein condensate interferometry. Phys. Rev. A, 72:051605(R), 2005.

[13] SJ Wu, YJ Wang, Q Diot, and M Prentiss. Splitting matter waves using an optimized standingwave light-pulse sequence. Phys. Rev. A, 71:043602, 2005.

[14] K J Hughes, B Deissler, J H T Burke, and C A Sackett. High-fidelity manipulation of a BoseEinstein condensate using an optical standing wave. Phys. Rev. A, 76:035601, 2007.

[15] J H T Burke, O Garcia, K J Hughes, B Livedalen, and C A Sackett. Compact implementation of a scanning transfer cavity lock. Rev. Sci. Instrum., 76:116105, 2005.

[16] M Olshanii and V Dunjko. Interferometry in dense nonlinear media and interaction-induced loss of contrast in microfabricated atom interferometers. arXiv: cond-mat/0505358, 2005.

[17] J Stickney, D Z Anderson, and A Zozulya. Increasing the coherence time of Bose-Einsteincondensate interferometers with optical control of dynamics. arXiv:physics/0701191, 2007. 
[18] M Olshanii. Atomic scattering in the presence of an external confinement and a gas of impenetrable bosons. Phys. Rev. Lett., 81:938, 1998.

[19] B Deissler, K J Hughes, J H T Burke, and C A Sackett. Measurement of the ac Stark shift with a guided matter-wave interferometer. physics:0709.4675v1, 2007.

[20] S R Segal, Q Diot, E A Cornell, M Prentiss, A A Zozulya, and D Z Anderson. On-chip BoseEinstein condensate interferometer with $0.5 \mathrm{~mm}$ arm length. In Bull. Am. Phys. Soc., volume 52, page 11. American Physical Society, 2007. 